\title{
The role of the microenvironment in tumor immune surveillance
}

\author{
Oluwadayo Oluwadara ${ }^{1}$, Luca Giacomelli ${ }^{2}$, Xenia Brant ${ }^{1,3}$, Russell Christensen ${ }^{4}$, \\ Raisa Avezova ${ }^{1}$, George Kossan ${ }^{1}$, Francesco Chiappelli ${ }^{1 *}$
}

\begin{abstract}
${ }^{1}$ School of Dentistry, Division of Oral Biology and Medicine; ${ }^{2}$ Tirrenian Stomatologic Institute, Lido di Camaiore (Lucca), Italy; ${ }^{3}$ CEO IPSEMG Belo Horizonte, Brazil; ${ }^{4}$ UCLA School of Dentistry, Division of Oral and Maxillofacial Pathology; Francesco Chiappelli - Email:
\end{abstract} fchiappelli@dentistry.ucla.edu; Phone: 310-794-6625; Fax: 310-794-7901; *Corresponding author

Received July 25, 2010; Accepted August 03, 2010; Published January 06, 2011

\begin{abstract}
:
The evidence appears compelling that the microenvironment, and associated biological cellular and molecular factors, may contribute to the progression of a variety of tumors. The effects of the microenvironment may directly influence the plasticity of T cell lineages, which was recently discussed (O’Shea \& Paul, 2010 [4]). To review the putative role of the microenvironment in modulating the commitment of tumor immune surveillance, we use the model of oral premalignant lesions.
\end{abstract}

Keywords: Oral lichen planus, oral squamous cell carcinoma, proteomic voting, tumor microenvironment, $\mathrm{T}$ cell lineages, tumor immune surveillance

\section{Background:}

The tumor microenvironment consists of paracrine factors, various cells, the stroma and its constituents. Compelling evidence suggests that it may contribute to the progression of various tumors [1, 2]. A better understanding of the roles of microenvironmental factors, and in particular the extent to which the microenvironment may modulate the plasticity of activities of tumor-surveilling immune cells, including the cytokines they produce, is advocated.

A variety of immune cells are involved in regulating the microenvironment in oral lichen planus (OLP), oral premalignant diseases (OPM), including oral epithelial dysplasia, and oral squamous cell carcinoma (OSCC) [3] These cells exert antitumor activities, but may also have a role in the inflammatory process and in the secretion of several cytokines with prooncogenic activity [3]

Here, we review the role of the microenvironment in the plasticity of tumor immune surveillance. We use OLP, OPM and OSCC as models to discuss the context of diseased tissues and their microenvironment. We focus on the role of T-cell signaling, the modulating effect of microenvironment factors on key signaling pathways, such as PI-3k, and examine several important unresolved questions that pertain to the putative role of the tumor microenvironment in the design of therapeutic approaches. Taken together, the evidence we discuss sheds new light on the importance of the microenvironment in the progression of OLP, OPM and OSCC. Successful management of these conditions in the near future may involve a coordinated early interventional treatment targeted towards the diseased tissues and their microenvironment, while taking full advantage of the malleability of $\mathrm{T}$ cell plasticity. Our novel perspective provides a promising premise for early interventional management of the microenvironment in a variety of tumors.

\section{$\mathrm{CD4}^{+}$plasticity and $\mathrm{T}$ cell signaling:}

$\mathrm{CD}^{+} \mathrm{T}$ cells play a central role in immune functions, in the etiology of allergy and autoimmune diseases, including OLP, and in tumor immune

ISSN 0973-2063 (online) 0973-8894 (print)

Bioinformation 5(7): 285-290 (2011) surveillance. The extensive plasticity of $\mathrm{T}$ cells was recently reviewed in Science [4]. In brief, the model counters the current dogma that $\mathrm{CD} 4^{+} \mathrm{T}$ cells yield distinct subsets of end-stage maturation that are uniquely capable of producing distinct sets of certain cytokine profiles. Rather, the novel model [4] proposes that $\mathrm{CD}^{+} \mathrm{T}$ cells alter their end-stage commitment in response to varying circumstances [4], putatively determined, at least in part, by the microenvironment. The model further argues that these cells can equally alter the pattern of their produced cytokines, and shift from, for example, a $\mathrm{T}_{\mathrm{H}} 17$ commitment to a $\mathrm{T}_{\mathrm{H}} 1$ or a $\mathrm{T}_{\mathrm{H}} 2$ or a Treg-specific pattern, in response to the demands dictated by the microenvironment [4]. The shift patterns ultimately affect changes and determine the functional role of these immune cells. For example, $\mathrm{T}_{\mathrm{H}} 17$ cells, the subset of $\mathrm{CD}^{+} \mathrm{T}$ cells that produces IL-17 has a critical role in autoimmunity, as well as in interconnection with other immune cells in the microenvironment [5], and tumor immunology. For these reasons, this cell population is targeted in cancer therapy protocols $[\mathbf{5 , 6 , 7 ]}$. It is expected that this model of plasticity shift in $\mathrm{T}$ cell patterns will directly impact on functions, such as those enumerated for $\mathrm{T}_{\mathrm{H}} 17$ cells.

The plasticity of conversion of $\mathrm{CD}^{+} \mathrm{T}$ populations results in a great potential diversity in cytokines profiles. If this $\mathrm{T}$ cell plasticity model is correct, then the question arises as to whether or not targeted therapeutic interventions may be designed that can effectively direct $\mathrm{T}$ cell subsets toward this or that pattern of cytokines to enhance, or to diminish certain physiological processes at the molecular level, and reduce pathological and proto-oncogenic events.

Evidence suggests that pro-inflammatory activities of immune cells may lead to cancer associated with chronic inflammation. Esophageal adenocarcinoma, for example, is associated with chronic esophagitis [3]. Colorectal cancer from inflammatory bowel disease and gastric cancer is associated with chronic infections from Helicobacter pylori [8].

Chronic inflammation resulting in cancer is also well established in the oral cavity. Evidence, albeit still controversial, indicates that OLP may 


\section{Bioinformation}

evolve, in certain cases, into OSCC [9]. Other OPM are also characterized by chronic inflammation, and could ultimately precipitate transformation [8]. The model of $\mathrm{CD}^{+} \mathrm{T}$ cells plasticity [4] begs the question, therefore of what might be the best immunotherapeutic approach to target these plurifaceted situations (Figure 1).

Case in point, OLP lesions are characterized with such $\mathrm{T}$ cell subset plasticity: the OLP pathology reflects, among others, both aspects of autoimmunity and associated $\mathrm{CD}^{+} \mathrm{T}$ cell involvement. Cytotoxic outcomes result from the concerted activities of $\mathrm{CD}^{+}$and $\mathrm{CD}^{+} \mathrm{T}$ cells on activated dendritic cells and apoptotic keratinocytes. It is possible and even probable that the activities of the $\mathrm{CD}^{+} \mathrm{T}$ cells in OLP are modulated by subsets of CD4+ T cells, themselves subject of plastic alterations driven by the microenvironment, which may determine the course of the pathology in each individual case. If this process obtains, we speculate that it might contribute to explain why certain OLP patients have high rate of recurrence with low remission, while others do not, while still others develop OSCC at the site of the OLP lesion. If the interplay between the microenvironment and $\mathrm{T}$ cell plasticity is indeed critical to the potential of OLP lesions for transformation, then the model may also shed light on proteomic-based intervention measures aimed at halting or slowing disease progression. T cell signaling and signaling generated by stroma cells and paracrine factors may further modulate $\mathrm{T}$ cell subset plasticity (Figure 1). The PI-3k pathway may play an important role in this context: we have shown that OLP biopsy samples from patients at high risk of cancer progression display increased expression of Lck, PI-3k and survivin [10].

$T$ cells and PI-3k signaling:

PI-3k signaling is critical in $\mathrm{T}$ cell activation and $\mathrm{T}$ cell migration. Inhibition of the PI-3k pathway has been invoked in $\mathrm{T}$ cell-mediated immunopathologies [11], including cases of transplant rejection, as well as autoimmune and inflammatory disorders. Furthermore, premalignancies and several cancers exhibit a derangement in the PI-3k pathway. Clinical trials of PI-3k inhibitors are ongoing $[12,13]$. T cell-mediated oral pathologies, including OSCC present an increased activity in the PI-3k pathway [14]

We speculate that the PI-3k pathway may play an important role in defining $\mathrm{T}$ cell plasticity, if only in part because PI-3k's modulation of T cell migration may contribute to slight (or important) changes in the microenvironment. Consequently, in the context of tumor immune surveillance, the overall impact of PI-3k signaling in $\mathrm{T}$ cells may be exerted both at the intra- and extracellular level. A case in point pertains to the reports that demonstrate that chemokine receptors (CXCR-4) and adhesion molecules (VLA-4 and CD44) are therapeutic targets in a leukemic microenvironment [15].

Taken together, these multiple threads of evidence lend support to the hypothesis that, in the treatment of leukemia for instance, molecular therapies must target both the leukemic cells and its microenvironment, since there appears to be both genetic and epigenetic aberrations in the stroma associated with leukemia [15]. We propose the argument that, since excessive PI-3k signaling is reputably one of the causes of leukemia, it is possible and even probable that a biological situation similar to that described in leukemia also manifests in OLP, OPM and OSCC. Consequently, as is the case in the treatment of leukemia, we suggest that there may be a place for PI-3k inhibitors in the therapeutic intervention of oral cancer, oral premalignant lesions, autoimmune and chronic inflammatory disorders, and OLP.

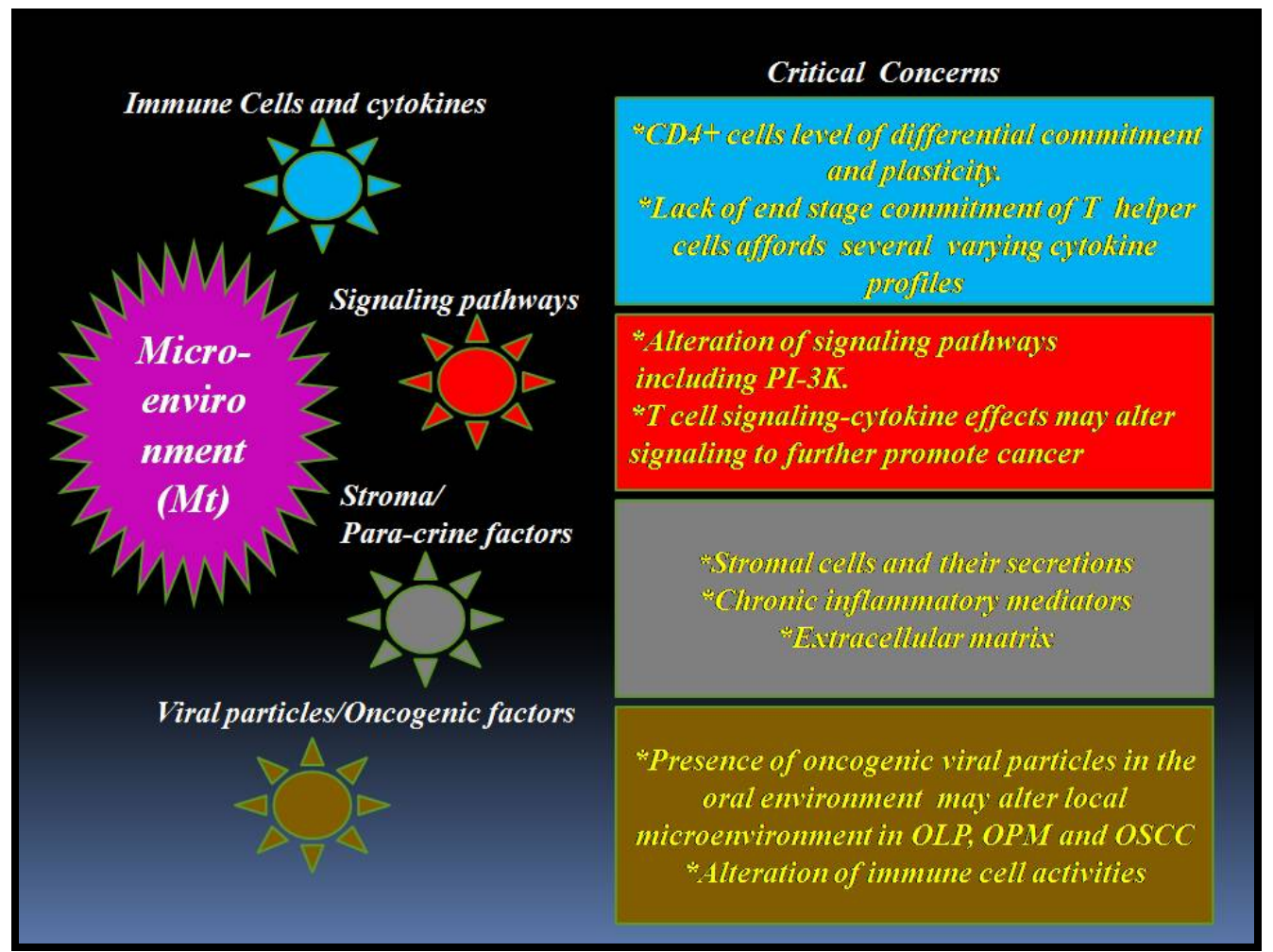

Figure 1: The multiple players in the microenvironment. Immune cells, cytokines, growth factors that mediate specific signaling pathways, stroma and paracrine factors, viral particles that may be oncogenic, and a myriad of additional biological factors actively compose the microenvironment. Based on the existing research evidence, we proffer the hypothesis that fine alterations in the balance of these components of the microenvironment distinguish OLP, OPM and SCCA. Changes in the microenvironment may contribute to the reported plasticity of CD4 ${ }^{+} \mathrm{T}_{\text {cell }}$ lineages, which itself results in altered cytokine profiles with associated important changes in the microenvironment that may drive significant alteration in the regulation of T-cell signaling events, including PI-3k, Lck, mTor, etc. 


\section{Bioinformation}

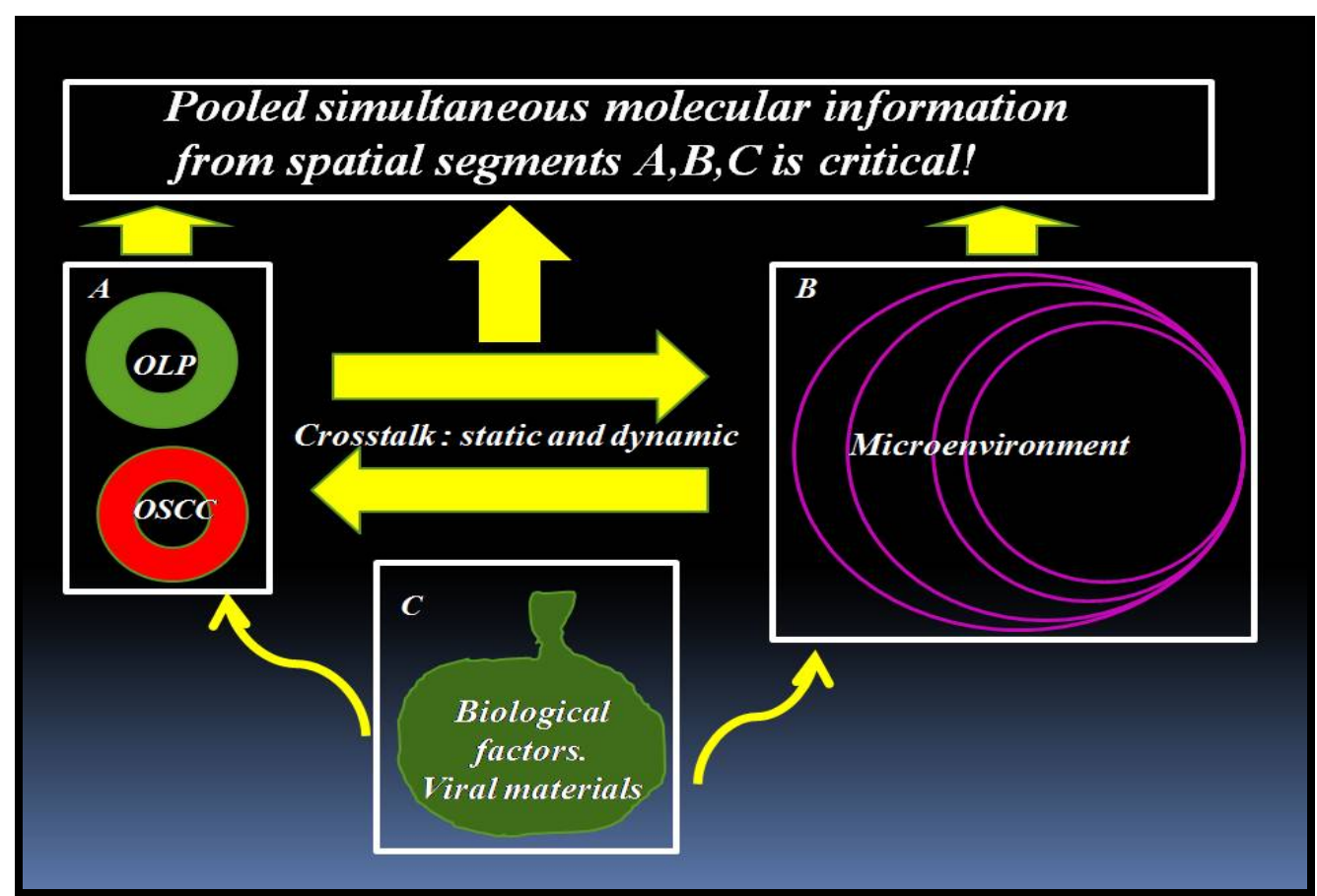

Figure 2: Pooled simultaneous molecular information from spatial segments is critical. The molecular spatial segments of the disease entity encompass the microenvironment and associated biological factors. A full realization of the complex nature of the microenvironment is critical to achieve personalized therapy: bringing together the molecular events in these spatial segments, drug micro-targeting modes of intervention will prevent drug resistance, and help predict possible treatment failure or success.

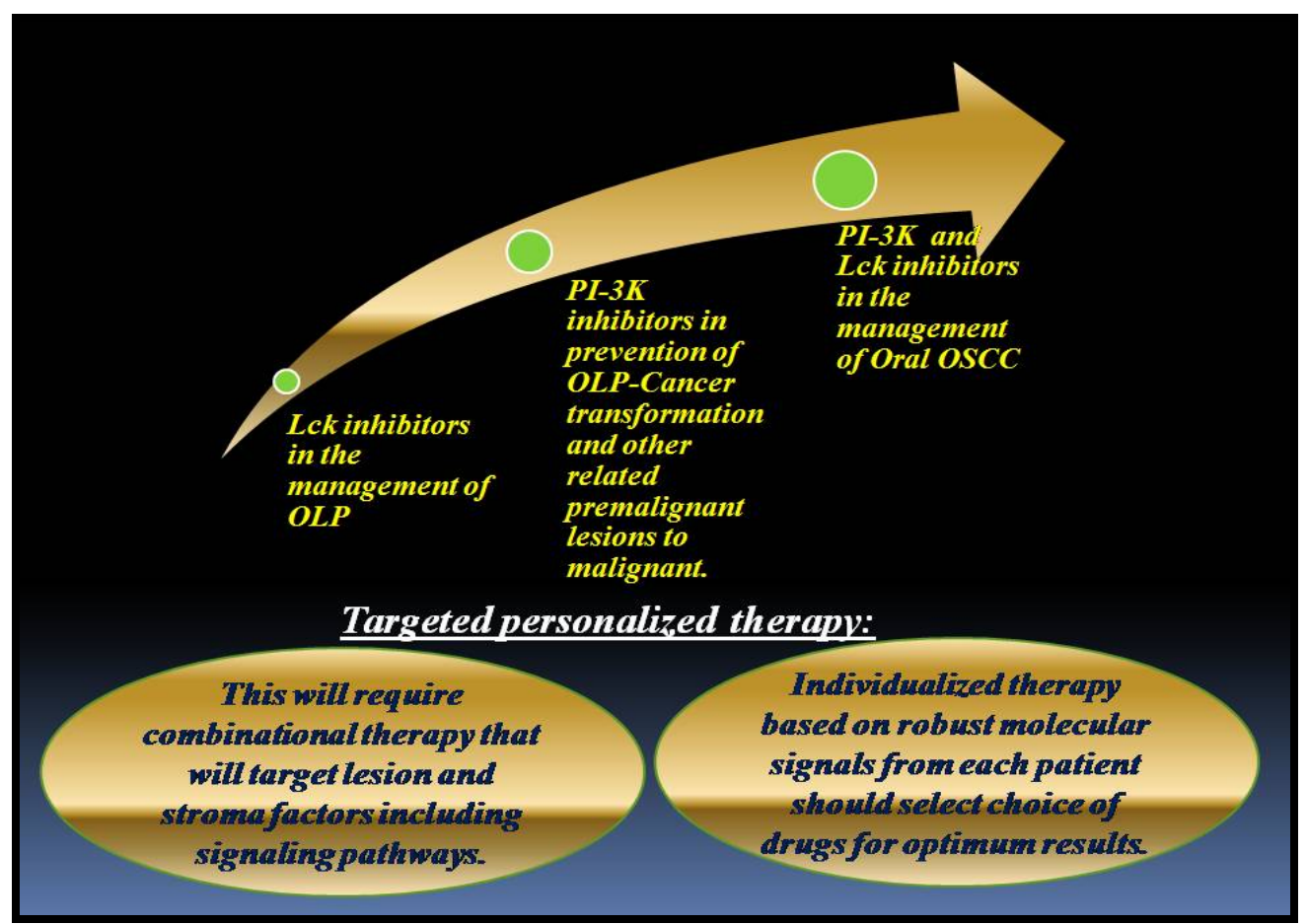

Figure 3: Lck inhibitors and PI-3k inhibitors in the management of OLP, OPM and OSCC. Lck is a src tyrosine kinase critical to T-cell signaling. PI-3k inhibitors target the PI-3k enzyme and inhibit its activities. Current clinical trials are ongoing to test PI-3k inhibitors. We postulate that Lck inhibitors will be found to be beneficial for OLP, particularly in combination with PI-3k inhibitors. We propose that such inhibitor cocktails will be beneficial for most cases of OLP, OPM and OSCC, because this combinational approach will target the disease itself (OLP, OPM and OSCC) as well as stroma factors in the microenvironment. 


\section{Bioinformation}

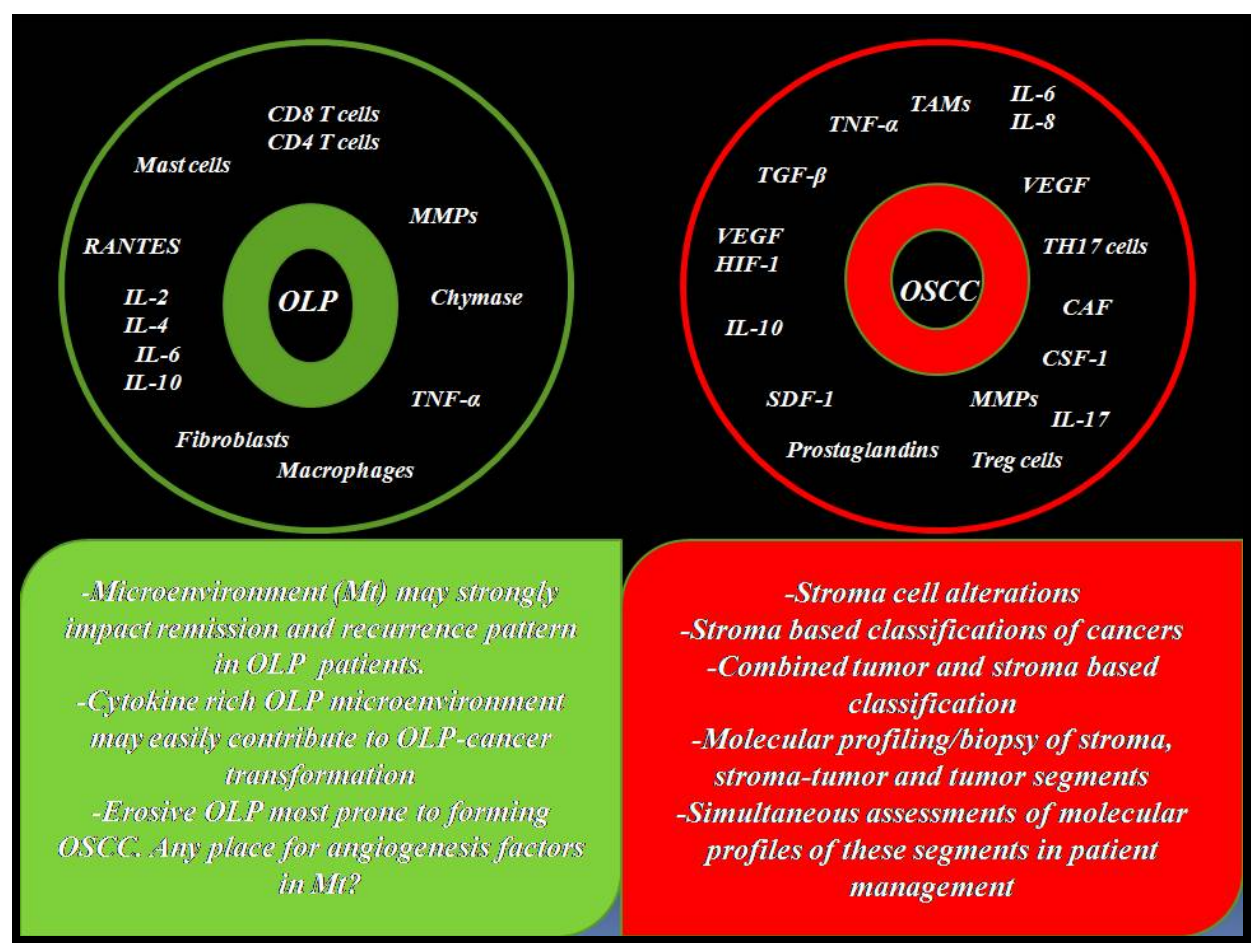

Figure 4: The profiles of cells and cytokines associated with microenvironment in OLP and OSCC. The profile of cytokines and cells in OLP and OSCC share few similarities. In OLP, the microenvironment has several interleukins (IL-2, 4, 6, 10), as well as CD4 ${ }^{+}$, CD8 ${ }^{+} \mathrm{T}$ cells, enzymes, and other cell populations associated with inflammation. In OSCC, enzymes, interleukins (IL-6, 8), inflammatory cells and mediators, angiogenesis factors (VEGF) contribute associated to the microenvironment. The microenvironment may strongly impact remission and recurrence patterns in OLP patients and the pattern of OLP-cancer transformation. Other than classification of tumors, stroma-based tumor classification may enhance combined tumor-stroma classification. Simultaneous assessments of microenvironment and these lesions should inform better correlation of molecular information.

\section{Methodology:}

Molecular mapping of microenvironment in OLP, OPM and OSCC:

Using breast cancer as a model, current molecular trends [16] indicate that the same cancer type may present distinct molecular signatures in different individuals. This observation dictates that effective modes of therapy must be tailored to each specific molecular signature.

Similar observations of distinct molecular signatures have been noted in oral lesions [17]. Hence, it is likely that reticular and erosive OLP lesions will be found to be characterized by distinct molecular signatures, which, putatively, will be determined in large part by, and may contribute to the architecture of the microenvironment. These alterations may contribute in explaining the reported increase rate of cancer transformation associated preferentially with erosive, compared to reticular OLP lesions [18]. Similar arguments stand for the increase rate of cancer transformation from the two distinct forms of OPM, epithelial dysplasia (EpD) and erythroplakia, compared to leukoplakia [19]. A case in point pertains to our data, which showed that OPM cases of EpD revealed similar molecular signatures with OSCC cases [10]

Taken together, these lines of evidence suggest that appropriate management of different oral pathologies (OLP, OPM, and OSCC) will be improved if the distinct physio-pathological dimensions of the microenvironment of the lesion per se will be considered; including associated biological and viral factors (Figure 2). While there might be a dynamic gradient of various cytokines or concentration of $\mathrm{CD}_{+}+\mathrm{T}$ cell subpopulations within the microenvironment of OLP, OPM, and OSCC, basic similarities and commonalities will exist, we anticipate, in these microenvironments.

The dynamics of the microenvironment is further complicated with direct communication between cancer cells and their microenvironment. Such direct communication is expected in OLP and OPM cases (Figure 2).

ISSN 0973-2063 (online) 0973-8894 (print)

Bioinformation 5(7): 285-290 (2011)
Early detection and prevention of transformation of OLP lesions will benefit from active studies of microenvironment, and better inform evidence-based therapeutic interventions.

Current studies on microenvironment have focused more on its impact on invasion and metastasis in cancer [20]. Evidence-based assessment for OLP therapy [21] has not revealed superior choice of drug in the management of this disease, which suggests that the future of personalized therapy will depend on the full and accurate molecular and proteomic description of the disease entity and associated microenvironment in each patient. This approach will only be possible in a translational evidencebased medicine context, with novel and improved technological tools to characterize the pathology of the microenvironment.

\section{Discussion:}

Important Unresolved Questions: The Putative Role of Proteomic Inhibitors?

Our recent work on molecular biomarker profiling [10] in OLP evaluated T cell signaling. Lck, a src tyrosine kinase, acts downstream of the TCR complex to regulate T-cell signaling. OLP biopsy samples show robust expression of Lck [10], which suggests that the chronic inflammatory nature of the microenvironment in OLP might be reduced by targeting $\mathrm{T}$ cell signaling, and specifically the pivotal role of Lck (Figure 3). Such therapeutic measures may curtail the impact of $\mathrm{T}$ cell plasticity and subsets signatures of cytokines, while minimizing inhibition of basic physiological activities.

Another similar approach is to multi-target key proteins in the $\mathrm{T}$ cell signaling pathways, such as those found at the level of the surface receptors or downstream to Lck, or those that act as co-stimulatory receptors. Multi-targeting may be advantageous, because it may preclude drug resistance, and be, overall, more potent by reducing or hindering 
signaling pathway crosstalk (Table 1 see Supplementary material). Multi-targeting ZAP-70, PI-3k, and nuclear transcription factors are alternate prime targets because these factors act downstream to Lck.

Trials of PI-3k inhibitors for cancer are ongoing [12, 13]. These interventions have not been tested in the context of the oral pathologies discussed here. However, based on the novel perspectives explored above [4] and our own data [10] we argue that PI-3k inhibitors may soon find effective therapeutic use in the treatment of premalignant oral lesions, including OLP. Considering the adoption of these drugs in the armamentarium for treating premalignant oral lesions will improve the current understanding of the molecular efficacy of these drugs, and their mechanisms of action as well as resistance, and ultimately lead to improved treatment.

Molecular proteomic profiling, such as our work [10], further indicates that OLP, OPM, OSCC and other oral and pharyngeal cancers may benefit from therapy based on inhibitors of PI-3k, Lck, and related signaling pathways, used as single or multi-drug therapy. It is our contention here that a principal mode of action of these interventions is to target specific events modulated by $\mathrm{T}$ cells subpopulations, and driven by the microenvironment, such as, for instance, the use of anti-Lck to control inflammation, cancer progression and metastasis, or the use of PI-3k inhibitors in modulating the microenvironment to subdue tumor transformation and proliferation in oral premalignant lesions such as epithelial dysplasia.

Our proteomic profiling studies indicate that other proteins in the PI-3krelated pathways may also be targeted, including Akt and mTor. Case in point, our data mining analysis suggested a critical role of Akt [22] in these disease entities. mTor acts downstream to Akt, and the mTor inhibitor rapamycin is already undergoing clinical development, with promising efficacy in the treatment of OLP lesions [23].

Another approach to intervene in OLP, OPM and OSCC may be to targe directly certain key factors of the microenvironment (Figure 4). Such direct targeting might require specific antibodies or decoy receptors. The fact that certain cytokines, immune cells and enzymes distinguish the microenvironments of the OLP and OSCC is a further confirmation of the similarity between these disease entities at the molecular and proteomic levels (Figure 4).

\section{Conclusion:}

Multi-factorial assessment informs multi-drug personalized targeting: Outlining the future:

With the advent of more reliable tools for molecular analysis (e.g., protein microarray, MALDI), the future management of disease entities such as those proposed here as models for our discussion (i.e., OLP, OPM, OSCC) can be based on the proteomic signatures of the lesion proper, and microenvironment analysis. Analysis of the latter will include stromabased classifications, cytokine assessment; in-cell western blot analysis for signaling pathways and laser capture micro-dissection (LCM). LCM is effective in living and non-living tissue types to obtain localized segments for analysis of DNA, RNA and protein [24]

This approach will provide better insights into the biology of OLP, OPM and OSCC, and it will allow a more robust global molecular outlook of a disease, than the current micro-targeting approach that exists (Figure 2). Current micro-targeting has resulted in an ineffective management of OLP lesions and in failure of cancer therapy, including drug resistance, and rapid progression of OPM cases to full blown cancer.
Personalized translational evidence-based medical therapy based on a characterization of the microenvironment and the model of $\mathrm{T}$ cell plasticity $[4,25]$ will follow the same global assessment disease state that we currently use. But, it will be supported with a more robust molecular picture of both the lesion proper, its microenvironment and related biological factors (Figure 2). Through the concept of biomarker voting $[10,26]$, the molecular pictures provided by these spatial segments can be correlated together for translational use. Taken together these correlated data will vary from patient to patient, but they will lead to fully informed translational evidence-based decision-making on treatment success, and afford better understanding for failure. The novel perspective we proffer will permit an easier identification and a better understanding of the complex network of biological factors that currently mask our management of these disease entities.

\section{Acknowledgements:}

The authors thank the University of California Senate, and the UCLA School of Dentistry for providing partial funding for this research.

References:

[1] MJ Bissell \& D Radisky, Nat Rev Cancer 1: 46 (2001) [PMID: 11900251]

[2] PA Kenny et al. Front Biosci 1: 3468 (2007) [PMID: 17485314]

[3] MD Mignogna et al. Oral Oncol 40: 120 (2004) [PMID: 14693234]

[4] JJ O'Shea \& WE Paul, Science 327: 1098 (2010) [PMID: 20185720]

[5] W Zou \& NP Restifo, Nat Rev Immunol 10: 248 (2010) [PMID: 20336152]

[6] M Beyer \& JL Schultze, Curr Pharm Des 15: 1879 (2009) [PMID: 19519430]

[7] JM Damsker et al. Ann N Y Acad Sci. 1183: 211 (2010) [PMID: 20146717]

[8] M Macarthur et al. Am J Physiol Gastrointest Liver Physiol. 286: G515 (2004) [PMID: 15010360]

[9] S Gandolfo et al. Oral Oncol 40: 77 (2004) [PMID: 14662419]

[10] Oluwadara et al. Bioinformation 4: 249 (2009)

[11] SG Ward \& FM Marelli-Berg, Biochem J. 418: 13 (2009) [PMID: 19159344]

[12] P Liu et al. Nat Rev Drug Discov. 8: 627 (2009) [PMID: 19644473]

[13] C Garcia-Echeverria \& WR Sellers, Oncogene 27: 5511 (2008) [PMID: 18794885]

[14] K Okkenhaug \& B Vanhaesebroeck, Nat Rev 3: 317 (2003) [PMID: 12669022]

[15] M Konopleva et al. Drug Resist Updat 12:103 (2009) [PMID: 19632887]

[16] RW Glenn, Ann Surg Oncol 17: 1392 (2010) [PMID: 20217261]

[17] AG Banerjee, Mol Cancer Ther 4: 865 (2005) [PMID: 15956244]

[18] M Fang et al. Quintessence Int 40: 235 (2009) [PMID: 19417887]

[19] SS Napier \& PM Speight, J Oral Pathol Med 37: 1 (2008) [PMID: 18154571]

[20] K Kessenbrock et al. Cell 141: 52 (2010) [PMID: 20371345]

[21] V Espina et al. Clin Lab Med 29: 1 (2009) [PMID: 19389547]

[22] L Giacomelli et al. Bioinformation 4: 258 (2009)

[23] A Soria et al. Dermatology 218: 22 (2009) [PMID: 18997452]

[24] D Farhi \& N Dupin, Clin Dermatol 28: 100 (2010) [PMID: 20082959]

[25] E Wohlfert \& Y Belkaid, Mucosal Immunol 3: 213 (2010) [PMID: 20237465]

[26] Oluwadara \& F Chiappelli, Bioinformation 3: 332 [PMID: 19707295]

Edited by F Chiappelli

Citation: Oluwadara et al. Bioinformation 5(7): 285-290 (2011)

License statement: This is an open-access article, which permits unrestricted use, distribution, and reproduction in any medium, for non-commercial purposes, provided the original author and source are credited. 


\section{Supplementary material:}

Table 1: Outlining the Future - Possible therapeutic approach

\begin{tabular}{|c|c|}
\hline & Possible therapeutic approach \\
\hline 1. & Inhibit key T cell signaling events (e.g., Lck). \\
\hline 2. & $\begin{array}{l}\text { Multi-target key proteins in the } \mathrm{T} \text { cell signaling pathway (e.g., downstream of Lck). Multi-targeting may preclude drug resistance, } \\
\text { reduce signaling pathways crosstalk and be more potent, and reveal novel proteomic profiles. }\end{array}$ \\
\hline 3. & Target co-stimulatory receptors. \\
\hline 4. & Inhibit PI-3k activity and targets downstream of PI-3k (e.g., mTor) \\
\hline 5. & Target the microenvironment, with specific antibodies and decoy receptors. \\
\hline 6. & $\begin{array}{l}\text { Multi-target proteins across signaling pathways-T cell signaling and PI-3k pathway. This type of combinatory and multi-targeted } \\
\text { therapeutic approach must rest on the best available research evidence and be personalized and to serve specifically each patient's } \\
\text { particular needs and situation, as determined by the microenvironment. In brief, the future of therapeutic approaches for OLP, OPM } \\
\text { and OSSC lies in translational evidence-based interventions. }\end{array}$ \\
\hline
\end{tabular}

